\title{
Clinical practice guidelines for the foot and ankle in rheumatoid arthritis: a critical appraisal
}

\author{
Kym Hennessy ${ }^{1,2^{*}}$, James Woodburn ${ }^{1}$, Martijn Steultjens ${ }^{1}$ \\ From Australasian Podiatry Council Conference 2013 \\ Sydney, Australia. 2-5 June 2013
}

\section{Background}

Clinical practice guidelines are recommendations systematically developed to assist clinical decision-making and inform healthcare. Many rheumatoid arthritis (RA) management guidelines are available. However, these guidelines under-represent the foot and ankle, even though foot and ankle problems are common. Guidelines must be high quality to be beneficial. This study aimed to identify and critically appraise clinical practice guidelines for foot and ankle management in RA.

\section{Methods}

Guidelines were identified electronically and through hand searching. Search terms 'rheumatoid arthritis', 'clinical practice guidelines' and related synonyms were used. Foot and ankle search terms were excluded, to ensure guidelines meeting the inclusion criteria were not precluded if foot and ankle management was not mentioned in the title or keywords. Critical appraisal and quality rating were conducted using the Appraisal of Guidelines for Research and Evaluation (AGREE) II instrument.

\section{Results}

Twenty-two guidelines were included. Five guidelines were high quality and recommended for use. Five high quality and six low quality guidelines were recommended for use with modifications. Six low quality guidelines were not recommended for use. Two guidelines were foot and ankle specific. Five early and eleven established RA guidelines were recommended for use. Five recommendation domains were identified in early and established RA guidelines. These were multidisciplinary team

care, foot healthcare access, foot health assessment/ review, orthoses/insoles/splints, and therapeutic footwear. Established RA guidelines also had an 'other treatments' domain.

\section{Conclusions}

Foot and ankle management for RA feature in most widely published clinical practice guidelines. Unfortunately, supporting evidence is low quality. Agreement levels are predominantly 'expert opinion' or 'good clinical practice'. Clinical practice guidelines require better underpinning by high quality research evidence. Clinical relevance: Identification of recommendations from high quality guidelines for podiatric care of RA related foot and ankle issues.

\section{Author details}

'Institute for Applied Health Research, School of Health \& Life Sciences, Glasgow Caledonian University, UK. ${ }^{2}$ Department of Podiatric Medicine, School of Science \& Health, University of Western Sydney, Australia.

Published: 31 May 2013

doi:10.1186/1757-1146-6-S1-015

Cite this article as: Hennessy et al:: Clinical practice guidelines for the foot and ankle in rheumatoid arthritis: a critical appraisal. Journal of Foot and Ankle Research 2013 6(Suppl 1):015.

\footnotetext{
* Correspondence: kym.hennessy@gcu.ac.uk

${ }^{1}$ Institute for Applied Health Research, School of Health \& Life Sciences,

Glasgow Caledonian University, UK

Full list of author information is available at the end of the article
} 